\title{
FOURIER SERIES WITH SMALL GAPS
}

\author{
P. ISAZA and D. WATERMAN
}

(Received 13 February 1987)

Communicated by J. F. Price

\begin{abstract}
A trigonometric series has "small gaps" if the difference of the orders of successive terms is bounded below by a number exceeding one. Wiener, Ingham and others have shown that if a function represented by such a series exhibits a certain behavior on a large enough subinterval $I$, this will have consequences for the behavior of the function on the whole circle group. Here we show that the assumption that $f$ is in any one of various classes of functions of generalized bounded variation on $I$ implies that the appropriate order condition holds for the magnitude of the Fourier coefficients. A generalized bounded variation condition coupled with a Zygmundtype condition on the modulus of continuity of the restriction of the function to $I$ implies absolute convergence of the Fourier series.
\end{abstract}

1980 Mathematics subject classification (Amer. Math. Soc.): 42 A 16, 42 A 55, 26 A 45.

\section{Introduction}

A trigonmetric series $\sum a_{n} \cos n x+b_{n} \sin n x=\sum A_{n}(x)$ is said to be lacunary if it exhibits large gaps, that is, $a_{n}=b_{n}=0$ except for $n \in\left\{n_{k}\right\}$ where $n_{k+1} / n_{k} \geq q>1, k=1,2, \ldots$. Such series have been extensively studied (Zygmund [10], Chapter V, Sections 6-8; Bary [1], Chapter XI) with results of the following character: some particular local property of the series implies that it has a certain global property.

(C) 1989 Australian Mathematical Society 0263-6115/89 \$A2.00+0.00 
We say that a trigonometric series $\sum A_{n_{k}}(x)$ has "small gaps" if $n_{k+1}-n_{k} \geq$ $q>1$. Although this case is briefly treated in the standard sources ([10], Chapter $\mathrm{V}, \S 9 ;[1]$, Chapter XI, §13), the literature is not well known and we will review it briefly.

These problems seem first to have been considered by Wiener [9], who showed that if a series with small gaps behaves well in a large enough interval, then some aspects of this behavior hold for $[0,2 \pi]$. His primary interest was the generalization of the Hadamard gap theorem.

Wiener's result was improved by Ingham [3] who employed it to establish a generalized form of the Hardy-Littlewood "high-indices" theorem. Kennedy [4] established results on nonharmonic trigonometrical series which specialize to the harmonic case as follows: If $n_{k+1}-n_{k} \rightarrow \infty$ and $\sum A_{n_{k}}(x)$ is the Fourier series of an integrable function $f$, then

(i) $f$ of bounded variation ( $B V)$ on an interval $I$ implies $a_{n}, b_{n}=O\left(n^{-1}\right)$.

(ii) $f \in \Lambda_{\alpha}$ on $I, 0<\alpha<1$, implies $a_{n}, b_{n}=O\left(n^{-\alpha}\right)$.

(iii) $f \in \Lambda_{\alpha}$ on $I, \frac{1}{2}<\alpha<1$, implies $\sum\left|a_{n}\right|+\left|b_{n}\right|<\infty$.

(iv) $f \in \Lambda_{\alpha}$ on $I, 0<\alpha<1$, and $f \in B V$ implies $\sum\left|a_{n}\right|+\left|b_{n}\right|<\infty$.

Here $\Lambda_{\alpha}$ denotes the Lipschitz class of order $\alpha$. Actually he showed these results hold if $\underline{\lim }\left(n_{k+1}-n_{k}\right)>32 \pi /|I|$ and, therefore hold regardless of the length of $I$ if $n_{k+1}-n_{k} \rightarrow \infty$.

Noble [5] had established the above conclusions under the assumption $\left(n_{k+1}-\right.$ $\left.n_{k}\right) / \log n_{k} \rightarrow \infty$. Bary [1], Chapter XI, $\S 13$, reports on the work of Noble and slight generalizations of it by Ul'yanov. Her comment to the effect that Kennedy required $f \in L^{2}([0,2 \pi])$ in his results is misleading. Patadia [6] has shown that a similar extension of Stečkin's theorem on absolute convergence ([1, page 196]) can be made by assuming the conditions of that theorem to hold on an arbitrary interval and requiring further $n_{k+1}-n_{k} \rightarrow \infty$.

\section{Definitions and background}

Let $f$ be a real function defined on the circle group $T([0,2 \pi))$. $\left\{I_{n}\right\}$ will denote a collection of nonoverlapping intervals in $T$. If $I=[a, b]$, then $f(I)=f(b)-f(a)$.

If $\Lambda=\left\{\lambda_{n}\right\}$ is a nondecreasing sequence of positive real numbers such that $\sum 1 / \lambda_{n}=\infty$, we say that $f$ is of $\Lambda$-bounded variation $(\Lambda B V)$ if $\sum_{1}^{\infty}\left|f\left(I_{n}\right)\right| / \lambda_{n}$ $<\infty$ for every $\left\{I_{n}\right\}$. This is known to imply that the collection of sums $\sum\left|f\left(I_{n}\right)\right| / \lambda_{n}$ is bounded [8].

Let $\varphi(x)$ be a nonnegative convex function defined on $[0, \infty)$ such that $\varphi(x) / x$ $\rightarrow 0$ as $x \rightarrow 0$. We say that $f$ is of $\varphi$-bounded variation $(\varphi B V)$ if for some $c>0$, $\sup \left\{\sum \varphi\left(c\left|f\left(I_{n}\right)\right|\right) \mid\left\{I_{n}\right\}\right\}=V_{c}(f)<\infty$. 
If $h(n)$ is a nondecreasing concave-downward function on the positive integers, we say that $f \in V[h]$ if there is a constant $C$ such that $\sum_{1}^{n}\left|f\left(I_{k}\right)\right| \leq C h(n)$, $n=1,2, \ldots$, for every collection $\left\{I_{n}\right\}$.

We say that $f$ is in one of the classes on $I \subset T$ if, in the definition, we restrict $\left\{I_{n}\right\}$ by $I_{n} \subset I$.

We concern ourselves here with the known estimates of the order of magnitude of the Fourier coefficients for functions in these classes [7] and with a condition for the absolute convergence of Fourier series of $V\left[n^{\alpha}\right][2]$, showing that these results hold if the conditions are satisfied on a (large enough) small interval $I \subset T$.

\section{Statement of results}

We suppose throughout that $f$ is a real function in $L^{1}(T)$ with Fourier series $\sum c_{n_{k}} e^{i n_{k} x}, \quad n_{-k}=-n_{k}$, satisfying $n_{k+1}-n_{k} \geq q>1, k=0,1,2, \ldots$ Let $I \subset T$ be a closed interval with length $|I|=(1+\delta) 2 \pi / q, \delta>0$.

We have the following results.

THEOREM 1. With $f$ and $I$ as above,

(i) $f \in V[h]$ on I implies $c_{n}=O(h(|n|) /|n|)$.

(ii) $f \in \Lambda B V$ on $I$ implies $c_{n}=O\left(1 / \sum_{1}^{|n|} 1 / \lambda_{i}\right)$.

(iii) $f \in \varphi B V$ on $I$ implies $c_{n}=O\left(\varphi^{-1}(1 /|n|)\right)$.

THEOREM 2. Let $f$ and $I$ be as above. Let $\omega_{I}(f, t)$ be the modulus of continuity of $f$ restricted to $I$. If $f \in V\left[n^{\alpha}\right]$ on $I, 0 \leq \alpha<\frac{1}{2}$, and

$$
\sum_{n=1}^{\infty} \frac{1}{n} \omega_{I}^{(1-2 \alpha) / 2(1-\alpha)}\left(f, \frac{1}{n}\right)<\infty,
$$

then the Fourier series of $f$ converges absolutely.

It is clear that if we make the assumption $n_{k+1}-n_{k} \rightarrow \infty$, then the conclusions hold for any nondegenerate interval $I$.

\section{Preliminaries}

The proofs of our theorems rest on two other results: 
LEMMA 1. Let $f \in L^{1}(T) \cap L^{2}(I), I$ a closed subinterval of $T$. The sequence of partial sums of the Fourier series of $f$ converges to $f$ in $L^{2}\left(I^{\prime}\right)$ for any closed interval $I^{\prime}$ in the interior of $I$.

Proof. With $I$ and $I^{\prime}$ as above, let $g=f$ on $I$ and $g=0$ on $T \backslash I$. Let $S_{n}(\cdot)$ be the $n$th partial sum of the Fourier series of a function. Since $g \in L^{2}(T)$ we have $\left\|S_{n}(g)-g\right\|_{L^{2}(T)} \rightarrow 0$ as $n \rightarrow \infty$. Therefore,

$$
\begin{aligned}
\left\|S_{n}(f)-f\right\|_{L^{2}\left(I^{\prime}\right)} & \leq\left\|S_{n}(f)-S_{n}(g)\right\|_{L^{2}\left(I^{\prime}\right)}+\left\|S_{n}(g)-f\right\|_{L^{2}\left(I^{\prime}\right)} \\
& \leq\left\|S_{n}(f)-S_{n}(g)\right\|_{L^{2}\left(I^{\prime}\right)}+\left\|S_{n}(g)-g\right\|_{L^{2}(T)} \\
& =o(1),
\end{aligned}
$$

since the localization principle implies that $S_{n}(f)-S_{n}(g) \rightarrow 0$ uniformly on $I^{\prime}$.

Lemma 1 enables us to extend a result due to Ingham [3] as follows.

LEMMA 2. Let $\sum c_{n_{k}} e^{i n_{k} x}$ be the Fourier series of a function $f \in L^{1}(T) \cap$ $L^{2}(I)$ where $-n_{k}=n_{-k}, n_{k+1}-n_{k} \geq q>1, k=0,1,2, \ldots$, and $|I|=(1+\delta) 2 \pi / q$ for some $\delta>0$. Then

$$
\sum_{-\infty}^{\infty}\left|c_{n_{k}}\right|^{2} \leq\left(A_{\delta} /|I|\right) \int_{I}|f(x)|^{2} d x
$$

where $A_{\delta}=2 \pi(1+\delta)^{2} / 4 \delta(2+\delta)$.

PROOF. Let $I_{\eta}$ be a closed interval of length $(1+\eta) 2 \pi / q$ concentric with $I$, $\eta<\delta$, and let $S_{N}(x)=\sum_{k=-N}^{N} c_{n_{k}} e^{i n_{k} x}$. Then by [10], Chapter V, Theorem 9.1

$$
\sum_{k=-N}^{N}\left|c_{n_{k}}\right|^{2} \leq\left(A_{\eta} /\left|I_{\eta}\right|\right) \int_{I_{\eta}}\left|S_{N}(x)\right|^{2} d x .
$$

By Lemma $1, S_{n} \rightarrow f$ in $L^{2}\left(I_{\eta}\right)$ as $N \rightarrow \infty$ and therefore $\int_{I_{\eta}}\left|S_{N}\right|^{2} \rightarrow \int_{I_{\eta}}|f|^{2}$, implying

$$
\sum_{-\infty}^{\infty}\left|c_{n_{k}}\right|^{2} \leq A_{\eta} /\left|I_{\eta}\right| \int_{I_{\eta}}|f(x)|^{2} d x
$$

Letting $\eta$ increase to $\delta$ we have the desired result.

\section{Proof of Theorem 1}

Let $|I|=2 \pi / q+2 \varepsilon$ and let $I^{\prime}$ be the concentric interval of length $2 \pi / q+\varepsilon$. Consider $|k|$ so large that $0<2 \pi /\left|n_{k}\right|<\varepsilon / 2$ and let $N=N_{k}=\left[\left(\left|n_{k}\right| \varepsilon / 4 \pi\right)-1\right]$. 
For $j=1, \pm 1, \pm 2, \ldots, \pm N$, let

$$
g_{j}(x)=f\left(x+2 \pi j /\left|n_{k}\right|+\pi / 2\left|n_{k}\right|\right)-f\left(x+2 \pi j /\left|n_{k}\right|-\pi / 2\left|n_{k}\right|\right) .
$$

Then, for every $s$,

$$
\hat{g}_{j}\left(n_{s}\right)=2 i c_{n_{s}} e^{i 2 \pi j n_{s} /\left|n_{k}\right|} \sin n_{s} \pi / 2\left|n_{k}\right| .
$$

(i) If $f \in V[h]$ on $I$, then $g_{j}$ is bounded on $I^{\prime}$ and, therefore $\sum_{-N}^{N} g_{j} \in L^{2}\left(I^{\prime}\right)$. By Lemma 2.

$$
\left|\sum_{j=-N}^{N} \hat{g}_{j}\left(n_{k}\right)\right|^{2} \leq \sum_{S=-\infty}^{\infty}\left|\sum_{j=-N}^{N} \hat{g}_{j}\left(n_{s}\right)\right|^{2} \leq C \int_{I^{\prime}}\left|\sum_{-N}^{N} g_{j}(x)\right|^{2} d x .
$$

(The letter $C$ will denote various constants independent of $k$.) Since $\hat{g}_{j}\left(n_{k}\right)=$ $2 i c_{n_{k}}$ sgn $n_{k}$ and $0<C \leq(2 N+1) /\left|n_{k}\right| \leq C^{\prime}$ for some constants $C$ and $C^{\prime}$ (that is, $\left.2 N+1 \sim\left|n_{k}\right|\right)$, it follows that

$$
\begin{aligned}
\left|c_{n_{k}}\right|^{2} & \leq \frac{C}{\left|n_{k}\right|^{2}} \int_{I^{\prime}}\left|\sum_{-N}^{N} g_{j}(x)\right|^{2} d x \\
& \leq \frac{C}{\left|n_{k}\right|^{2}} \int_{I^{\prime}}\left(\sum_{-N}^{N}\left|g_{j}(x)\right|\right)^{2} d x \\
& \leq \frac{C}{\left|n_{k}\right|^{2}}(h(2 N+1))^{2} \\
& \leq \frac{C}{\left|n_{k}\right|^{2}}\left(h\left(\left|n_{k}\right|\right)\right)^{2}
\end{aligned}
$$

which establishes (i).

(ii) If $f \in \Lambda B V$ on $I$ we observe that

$$
\left(\sum_{j=-N}^{N}\left|g_{j}(x)\right|\right)\left(\sum_{i=1}^{2 N+1} 1 / \lambda_{j}\right) \leq C(2 N+1)
$$

Thus from $(*)$ we have

$$
\left|c_{n_{k}}\right| \leq \frac{C}{\left|n_{k}\right|}(2 N+1) / \sum_{j=1}^{2 N+1} 1 / \lambda_{j} \leq C / \sum_{i}^{\left|n_{k}\right|} 1 / \lambda_{j} .
$$

This establishes (ii).

(iii) We observe first that for small enough $\alpha>0$ (independent of $k$ ),

$$
\varphi\left(\frac{1}{2 N+1} \sum_{-N}^{N} \alpha c\left|g_{j}(x)\right|\right) \leq \frac{\alpha}{2 N+1} V_{c}(f)<1 /\left|n_{k}\right|
$$


implying

$$
\sum_{-N}^{N}\left|g_{j}(x)\right|<C(2 N+1) \varphi^{-1}\left(1 /\left|n_{k}\right|\right)
$$

Thus, from $(*)$ we have

$$
\left|c_{n_{k}}\right| \leq \frac{C}{\left|n_{k}\right|}(2 N+1) \varphi^{-1}\left(1 /\left|n_{k}\right|\right)
$$

which yields (iii).

\section{Proof of Theorem 2}

Let $f \in V\left[n^{\alpha}\right]$ on $I$ and let $g_{j}$ and $I^{\prime}$ be as in the proof of Theorem 1 .

From Lemma 2 we have

$$
\sum_{s=-\infty}^{\infty}\left|\hat{g}_{j}\left(n_{s}\right)\right|^{2}=\sum_{s=-\infty}^{\infty} 4\left|c_{n_{s}}\right|^{2} \sin ^{2} n_{s} \pi / 2\left|n_{k}\right| \leq C \int_{I^{\prime}}\left|g_{j}(x)\right|^{2} d x
$$

for $j=0, \pm 1, \ldots, \pm N$. For $s$ such that $\left|n_{k}\right| / 2<\left|n_{s}\right| \leq\left|n_{k}\right|$, we have

$$
\sin ^{2} n_{s} \pi / 2\left|n_{k}\right| \geq \frac{1}{2}
$$

letting $\sum^{*}$ indicate summation over these values of $s$,

$$
\sum^{*}\left|c_{n_{\theta}}\right|^{2} \leq C \int_{I^{\prime}}\left|g_{j}(x)\right|^{2} d x
$$

Summing over $j$ and noting that $2 N+1 \sim\left|n_{k}\right|$ we have

$$
\sum^{*}\left|c_{n_{s}}\right|^{2} \leq \frac{C}{\left|n_{k}\right|} \int_{I^{\prime}} \sum_{-N}^{N}\left|g_{j}(x)\right|^{2} d x
$$

Fix $x \in I^{\prime}$ and for each integer $m \geq 0$, let

$$
E_{m}=\left\{j \mid 2^{-(m+1)} \omega_{I}\left(f, \pi /\left|n_{k}\right|\right)<g_{j}(x) \leq 2^{-m} \omega_{I}\left(f, \pi /\left|n_{k}\right|\right)\right\}
$$

Since $\left|g_{j}(x)\right| \leq \omega_{I}\left(f, \pi /\left|n_{k}\right|\right)$, each $j$ belongs to one and only one $E_{m}$. Let $\sigma_{m}$ be the cardinality of $E_{m}$. Since $f \in V\left[n^{\alpha}\right]$ on $I$,

$$
\sigma_{m} 2^{-(m+1)} \omega_{I}\left(f, \pi /\left|n_{k}\right|\right) \leq \sum_{j \in E_{m}}\left|g_{j}(x)\right| \leq C \sigma_{m}^{\alpha}
$$

implying

$$
\sigma_{m} \leq C 2^{m /(1-\alpha)} \omega_{I}^{-1 /(1-\alpha)}\left(f, \pi /\left|n_{k}\right|\right)
$$


Therefore

$$
\begin{aligned}
\sum_{j=-N}^{N}\left|g_{j}(x)\right|^{2} & =\sum_{m} \sum_{j \in E_{m}}\left|g_{j}(x)\right|^{2} \leq \sum_{m} \sigma_{m} 4^{-m} \omega_{I}^{2}\left(f, \pi /\left|n_{k}\right|\right) \\
& \leq C \sum_{m} 2^{-m(1-2 \alpha) /(1-\alpha)} \omega_{I}^{(1-2 \alpha) /(1-\alpha)}\left(f, \pi /\left|n_{k}\right|\right) \\
& \leq C \omega_{I}^{(1-2 \alpha) /(1-\alpha)}\left(f, \pi /\left|n_{k}\right|\right)
\end{aligned}
$$

implying

$$
\sum^{*}\left|c_{n_{s}}\right|^{2} \leq \frac{C}{\left|n_{k}\right|} \omega_{I}^{(1-2 \alpha) /(1-\alpha)}\left(f, \pi /\left|n_{k}\right|\right) .
$$

For large $m$, if there is $n_{s}$ such that $2^{m}<n_{s} \leq 2^{m+1}$, let $k=\max \left\{s \mid n_{s} \leq 2^{m+1}\right\}$. For such an $m$,

$$
\begin{aligned}
\sum_{2^{m}<\left|n_{s}\right| \leq 2^{m+1}}\left|c_{n_{s}}\right|^{2} & \leq \sum^{*}\left|c_{n_{s}}\right|^{2} \leq \frac{C}{\left|n_{k}\right|} \omega_{I}^{(1-2 \alpha) /(1-\alpha)}\left(f, \pi /\left|n_{k}\right|\right) \\
& \leq C 2^{-m} \omega_{I}^{(1-2 \alpha) /(1-\alpha)}\left(f, \pi / 2^{m}\right)
\end{aligned}
$$

Hence,

$$
\sum_{2^{m}<\left|n_{s}\right| \leq 2^{m+1}}\left|c_{n_{s}}\right| \leq C \omega_{I}^{(1-2 \alpha) / 2(1-\alpha)}\left(f, \pi / 2^{m}\right) .
$$

The theorem follows then from the fact that the convergence of $\sum_{-\infty}^{\infty}\left|c_{n_{s}}\right|$ is implied by the convergence of

$$
\sum_{0}^{\infty} \omega_{I}^{(1-2 \alpha) / 2(1-\alpha)}\left(f, \pi / 2^{m}\right)
$$

and the convergence of this last series is equivalent to that of

$$
\sum_{n=1}^{\infty} \frac{1}{n} \omega_{I}^{(1-2 \alpha) / 2(1-\alpha)}\left(f, \frac{1}{n}\right) \text {. }
$$

\section{References}

[1] N. K. Bary, A treatise on trigonometric series, Vol II (Macmillan, New York, 1964).

[2] Z. A. Čanturija, 'On the absolute convergence of Fourier series of the classes $V\left[n^{\alpha}\right]$ ', Fourier Analysis and Approximation Theory, Proc. Colloq., Budapest, 1976, Vol I., pp. 219-240 (Colloq. Math. Soc. János Bolyai 19, North-Holland, Amsterdam, 1978).

[3] A. E. Ingham, 'Some trigonometric inequalities with applications to the theory of series', Math. Z. 41 (1936), 367-379.

[4] P. B. Kennedy, 'Fourier series with gaps', Quart. J. Math. Oxford Ser. (2) 7 (1956), 224230. 
[5] M. E. Noble, 'Coefficient properties of Fourier series with gap conditions', Math. Ann. 128 (1954), 55-62.

[6] J. R. Patadia, 'On the absolute convergence of a lacunary Fourier series', J. Math. Anal. Appl. 65 (1978), 391-398.

[7] M. Schramm and D. Waterman, 'On the magnitude of Fourier coefficients', Proc. Amer. Math. Soc. 85 (1982), 407-410.

[8] D. Waterman, 'On $\Lambda$-bounded variation', Studia Math. 57 (1976), 33-45.

[9] N. Wiener, 'A class of gap theorems', Annali di Pisa 3 (1934), 367-372.

[10] A. Zygmund, Trigonometric series, 2nd ed. (Cambridge Univ. Press, New York, 1959).

Department of Mathematics

Syracuse University

Syracuse, New York 13244-1150

U.S.A. 